OPEN ACCESS

Edited by:

Xiaohui Li,

Shaanxi Normal University, China

Reviewed by:

Yang Yue,

Nankai University, China

Guobin Ren,

Beijing Jiaotong University, China Yongkang Gong,

Cardiff University, United Kingdom

*Correspondence:

Yana Shang

ynshang@shu.edu.cn

Specialty section:

This article was submitted to Optics and Photonics,

a section of the journal

Frontiers in Physics

Received: 17 March 2020

Accepted: 04 May 2020

Published: 03 June 2020

Citation:

Xu L, Shang Y, Yang J, Chen Z,

Pang F, Liu H, Dong Y, Chen N, Wen J and Wang $T$ (2020) Orbital Angular Momentum Optical Amplifier Based on PbS-Doped Ring-Core Fiber. Front. Phys. 8:198.

doi: 10.3389/fphy.2020.00198

\section{Orbital Angular Momentum Optical Amplifier Based on PbS-Doped Ring-Core Fiber}

\author{
Lingmin Xu, Yana Shang*, Jinhong Yang, Zhenyi Chen, Fufei Pang, Huanhuan Liu, \\ Yanhua Dong, Na Chen, Jianxiang Wen and Tingyun Wang
}

Key Laboratory of Specialty Fiber Optics and Optical Access Networks, Joint International Research Laboratory of Specialty Fiber Optics and Advanced Communication, Shanghai Institute for Advanced Communication and Data Science, Shanghai University, Shanghai, China

A unique orbital angular momentum (OAM) optical amplifier based on a ring-core fiber doped with lead sulfide (PbS) is realized for the first time in this study. The PbS-doped ring-core fiber is fabricated using the modified chemical vapor deposition method. The cross-sectional microscope image of the fiber is obtained. The difference in the refractive index between the ring core and the fiber cladding measured by a three-dimensional refractive index tester reaches $1 \%$. The sulfur and lead content in the ring-core of the $\mathrm{PbS}$-doped ring-core fiber is confirmed by energy spectrum analysis. A first-order OAM optical amplifier is built based on the PbS-doped ring-core fiber. The experiment confirms that the air-hole PbS-doped ring-core fiber can support the OAM $\mid /=1$ modes. In addition, $2.3 \mathrm{~dB}$ of on-off gain is obtained for the OAM $\mid /=1$ modes at $1,550 \mathrm{~nm}$. The fluorescence spectrum obtained using the backward pumping method ranges from 1,100 to $1,600 \mathrm{~nm}$.

Keywords: optical fiber amplifier, PbS-doped ring-core fiber, orbital angular momentum (OAM), on-off gain, fluorescence properties

\section{INTRODUCTION}

Mode division multiplexing (MDM) can be used to considerably expand the capacity of optical communications by using orthogonal modes to transmit signals $[1,2]$. In 2004, a new MDM communication scheme based on the photon orbital angular momentum (OAM) was proposed [3]. The initial studies had focused on the transmission of OAM modes in free space and suffered from short transmission distances. Since 2009, the index separation between adjacent OAM vector modes has been considerably enhanced by ring-core optical fiber structures [4-10]. In 2013, Bozinovic et al. [11] implemented OAM mode multiplexing in a ring-core optical fiber with a length of more than $1.1 \mathrm{~km}$. This shows that OAM can provide additional options for data multiplexing in future fiber optic networks. A variety of OAM amplifiers based on erbium-doped fibers have been theoretically designed [12-14] to address the unavoidable signal-transmission loss in long-distance communications. In the year 2017, Jung et al. [15] built the air-hole erbium-doped fiber amplifier (EDFA) to amplify the OAM $|l|=1$ modes for the first time. The gain of the OAM $|l|=1$ modes with a cladding pump was up to $15.7 \mathrm{~dB}$ for the wavelength ranging from 1,545 to 1,600 $\mathrm{nm}$. Xing et al. [16] used the erbium-doped few-mode fiber to build the all-fiber amplifier and realized the first-order OAM mode amplification in the year 2018, and the gain was $37.2 \mathrm{~dB}$.

However, the bandwidth of EDFA cannot cover the entire communication band of the $\mathrm{OH}$-free silica fiber. Therefore, an alternative active material is proposed for optical fiber amplifiers [17-19]. 
Because lead-sulfide $(\mathrm{PbS})$ quantum dots have strong quantumconfined effects, the luminescence center of $\mathrm{PbS}$ quantum dots ranges from 1,000 to $1,800 \mathrm{~nm}$, which is sufficient to realize the tunable photoluminescence in the near-infrared [20, 21]. $\mathrm{PbS}$ nanomaterials have great application prospects in optical amplifiers, lasers [22, 23], and sensors. The optical-gain performance has been observed in a glass medium doped using $\mathrm{PbS}$ quantum dots $[24,25]$. The amplification in $\mathrm{PbS}$ quantum dot-doped fibers in common communication bands has been considerably enhanced through improvements in various $\mathrm{PbS}$ based doping methods [26-30]. In the year 2018, Huang et al. [31] presented a new method, named "melt-in-tube," for preparing $\mathrm{PbS}$ quantum dot doped fiber. The $\mathrm{PbS}$ quantum dots with diameters from 1.0 to $5.0 \mathrm{~nm}$ were uniformly precipitated in the $\mathrm{PbS}$ quantum-dots doped glass fiber core. However, the fiber loss was considerable, i.e., $>14 \mathrm{~dB} / \mathrm{m}$.

In this study, we realize the amplification of the first-order OAM, which is based on an air-hole PbS-doped ring-core fiber. The fiber is fabricated using the modified chemical vapor deposition (MCVD) technology. The fluorescence spectrum ranges from 1,100 to $1,600 \mathrm{~nm}$. Under the pumping of a $980 \mathrm{~nm}$ laser, the on-off gain (up to $2.3 \mathrm{~dB}$ ) of the OAM $|l|=1$ modes with a signal light at $1,550 \mathrm{~nm}$ could be achieved.

\section{DESIGN AND FABRICATION OF AIR-HOLE PBS-DOPED FIBER}

\section{Preparation of Optical Fiber}

A considerable number of studies and experiments have shown that the effective index separation of OAM modes should be $>10^{-4}$ to support OAM mode transmission in the long haul. This can be realized by introducing a high index ring into the traditional fiber structure [5].

In this study, $\mathrm{PbS}$ powder is doped into the ring core of the fiber to support the transmission and amplification of OAM modes. An air-hole ring-core fiber doped using $\mathrm{PbS}$ is fabricated via MCVD. The process involved the following steps: First, $\mathrm{SiCl}_{4}$ and $\mathrm{GeCl}_{4}$ are carried by high-purity oxygen into a silica substrate tube, along with $\mathrm{PbS}$, which is vaporized by hightemperature heating. Second, the porous soot layer is deposited on the inner surface of the substrate tube. Third, at a high temperature of $2,000^{\circ} \mathrm{C}$, the silica substrate tube is disposed via a vitrification process and collapsed into a PbS-doped optical fiber preform. Finally, the preform is drawn into an air-hole $\mathrm{PbS}$-doped ring-core fiber with certain geometric parameters by using a drawing tower. During the drawing process, the structural parameters of the optical fiber can be controlled by the temperature of drawing, the preform feeding speed and the drawing speed.

The cross-sectional image of the fabricated fiber is observed via optical microscopy, and the refractive index profile of the fiber can be measured using a three-dimensional refractiveindex tester (Model: SHR-1602, Shanghai University, China). As depicted in Figure 1, the interior of the ring has a diameter of $9.80 \mu \mathrm{m}$ and is filled with air. The external diameter of the ring is $20.49 \mu \mathrm{m}$, and the thickness of the ring is $5.35 \mu \mathrm{m}$. In addition,

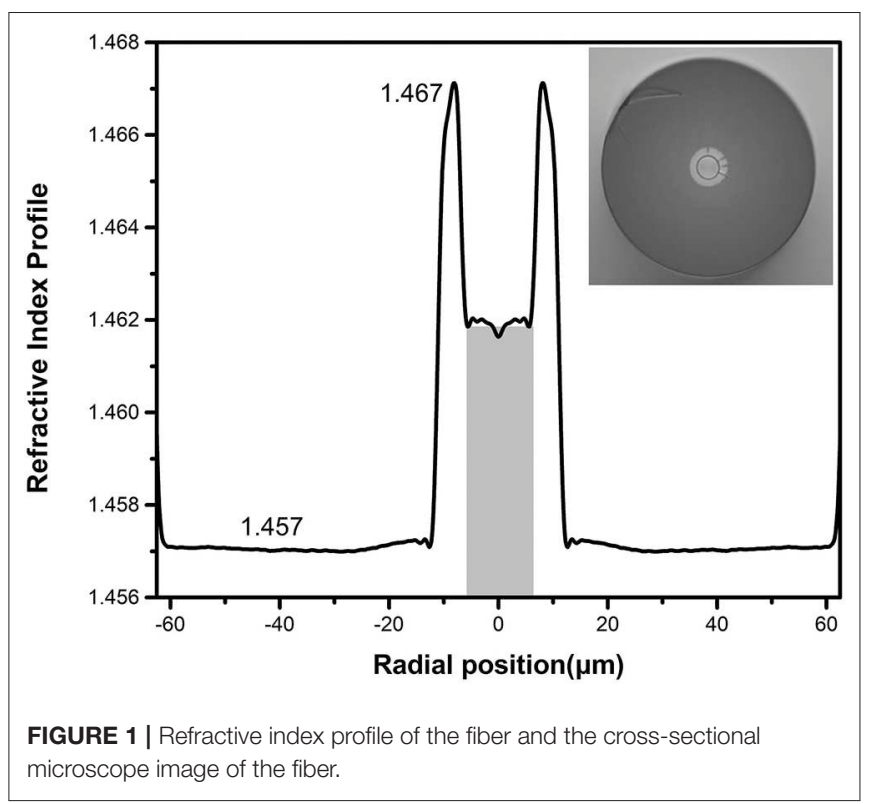

the diameter of the cladding is $130.22 \mu \mathrm{m}$. The refractive-index difference between the ring and cladding of the fiber reaches 1\% (the gray part in Figure 1 represents the air hole filled using index-matching oil). The total length of the sample fiber is about $60 \mathrm{~m}$. The loss of the fiber at $1,500-1,570 \mathrm{~nm}$ is about $19 \mathrm{~dB}$.

\section{Mode Simulation}

The RF module of COMSOL software is used to simulate the waveguide mode of optical fiber. The amplitude of the field decays rapidly as a function of the cladding radius. The cladding radius of the sample fiber is large enough for the field of the confined modes to reach zero at the exterior boundaries. This constitutes a valid boundary condition. Parameters such as the refractive index, cladding radius, ring core radius, and air hole radius of the sample optical fiber are set in the simulation. In addition, we use triangular grids to divide each part of the optical fiber. The region of air hole, ring core and cladding are divided into 148,230 , and 2,028 grids, respectively. Thus, the $\mathrm{HE}_{11}$, $\mathrm{TE}_{01}, \mathrm{TM}_{01}, \mathrm{HE}_{21}, \mathrm{HE}_{31}, \mathrm{EH}_{11}, \mathrm{HE}_{51}$, and $\mathrm{EH}_{31}$ vector modes transmitted by the fabricated $\mathrm{PbS}$-doped ring-core fiber can be analyzed using the COMSOL software. The effective indexes of the modes are listed in Table 1 . The effective index separation of the OAM $|l|=1$ modes is $0.65 \times 10^{-4}$, via calculation.

\section{CHARACTERIZATION ANALYSIS AND FLUORESCENCE SPECTRUM OF OPTICAL FIBER}

Energy-spectrum analysis is used to prove that the $\mathrm{PbS}$ material is successfully doped in the fiber. The element content in the three points indicated on the high-index ring depicted in Figure 2 are analyzed and the results are shown in Table 2 . It can be seen that in each of the selected regions, there exist the elements of $\mathrm{Si}$, $\mathrm{O}, \mathrm{C}, \mathrm{Pb}, \mathrm{S}$, and $\mathrm{Ge}$, thereby indicating that $\mathrm{PbS}$ is successfully 
TABLE 1 | Effective index.

\begin{tabular}{lr}
\hline Vector mode & Effective index \\
\hline $\mathrm{HE}_{11}$ & 1.462554 \\
$\mathrm{TE}_{01}$ & 1.462229 \\
$\mathrm{TM}_{01}$ & 1.462363 \\
$\mathrm{HE}_{21}$ & 1.462298 \\
$\mathrm{EH}_{11}$ & 1.461539 \\
$\mathrm{HE}_{31}$ & 1.461546 \\
$\mathrm{HE}_{51}$ & 1.458627 \\
$\mathrm{EH}_{31}$ & 1.458637
\end{tabular}

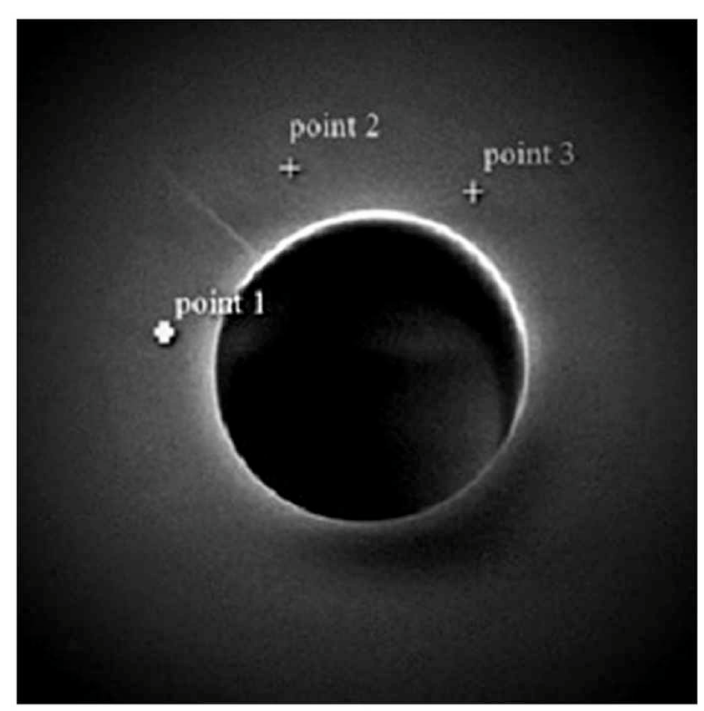

FIGURE 2 | Cross-sectional scanning electron microscope image of the optical fiber.

TABLE 2 | Element content analysis results.

\begin{tabular}{llll}
\hline Element & Point 1 (Wt\%) & Point 2 (Wt\%) & Point 3 (Wt\%) \\
\hline $\mathrm{O}$ & 45.23 & 45.11 & 44.86 \\
$\mathrm{Si}$ & 43.81 & 43.26 & 43.33 \\
$\mathrm{Ge}$ & 10.15 & 10.55 & 10.34 \\
$\mathrm{~Pb}$ & 0.65 & 0.67 & 1.16 \\
$\mathrm{~S}$ & 0.16 & 0.41 & 0.31 \\
total & 100.00 & 100.00 & 100.00 \\
\hline
\end{tabular}

doped into the air-hole ring-core fiber. However, the contents of elements $\mathrm{Pb}$ and $\mathrm{S}$ are different in different regions, indicating that the doping concentration of the $\mathrm{PbS}$ material is not uniform during the fiber-fabrication process.

The fluorescence spectrum of the optical fiber is measured using the backward pumping method. A $980 \mathrm{~nm}$ laser is selected as the pump light source. Figure 3 shows the fluorescence spectra of the fiber measured using an optical spectrum analyzer under various pump powers ranging from 100 to $700 \mathrm{~mW}$. The

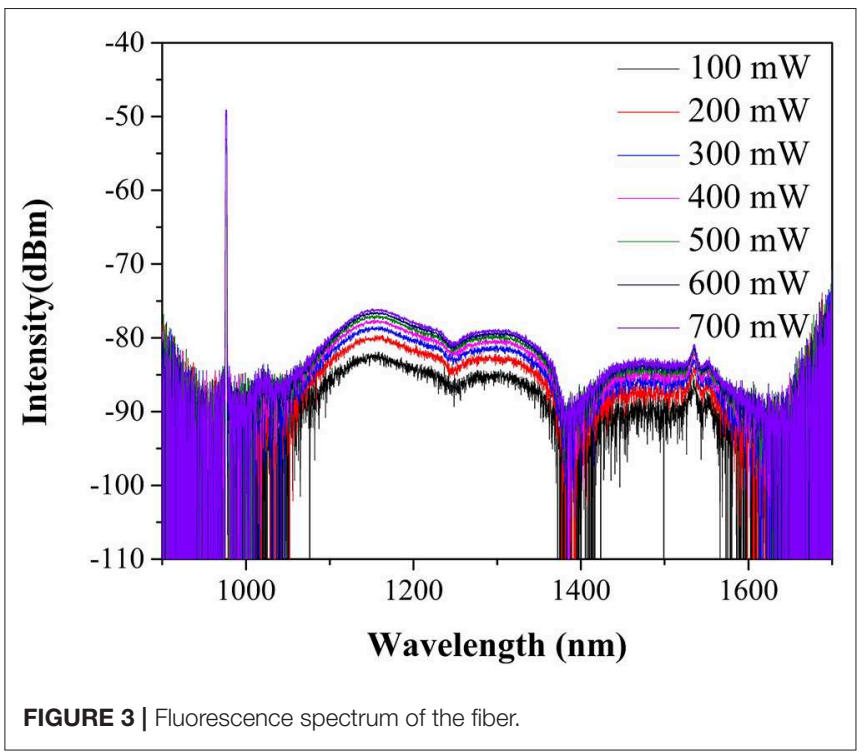

fluorescence spectra ranges from 1,100 to $1,600 \mathrm{~nm}$. In addition, the fluorescence intensity gradually increases with the pump power. The fluorescence intensity increases by only a small amount when the pump power increases from 600 to $700 \mathrm{~mW}$, and is almost unchanged and tends toward saturation when the pump power is further increased.

\section{OAM MODE GENERATION AND AMPLIFICATION}

An amplification system for the OAM $|l|=1$ modes is built using the fabricated fiber. The experimental setup is depicted in Figure 4. The Gaussian signal light is split into two beams using a $1 \times 2$ coupler. The first beam serves as an interference spherical reference beam. The second beam is converted into a vortex beam using a spiral phase plate. Both the signal light and pump light are coupled into the fabricated fiber using the DM. The OAM $|l|=1$ modes are successfully amplified subsequently. In addition, the beam profiles of the first-order vortex beam and the interference could be observed using an infrared camera. The DM before the infrared camera is used to filter out the pump light.

The beam profile of the OAM $|l|=1$ modes before entering the optical fiber input is depicted in Figure 5A, and their interference pattern in Figure 5B. The beam profile and the interference after exiting the output side of the air-hole PbSdoped ring-core fiber are depicted in Figures 5C,D, respectively. The interference with the spherical reference wave in Figure 5B proves that the OAM $|l|=1$ modes are successfully generated by the spiral phase plate. The beam profile maintained the form of a complete ring after exiting the output side of the fiber, as depicted in Figure 5C. A dextral spiral interference fringe is formed when the beam exiting the output side of the fiber interfered with the spherical reference beam, as depicted in Figure 5D. It indicates that the optical fiber could successfully support the $|l|=1$ OAM modes. 


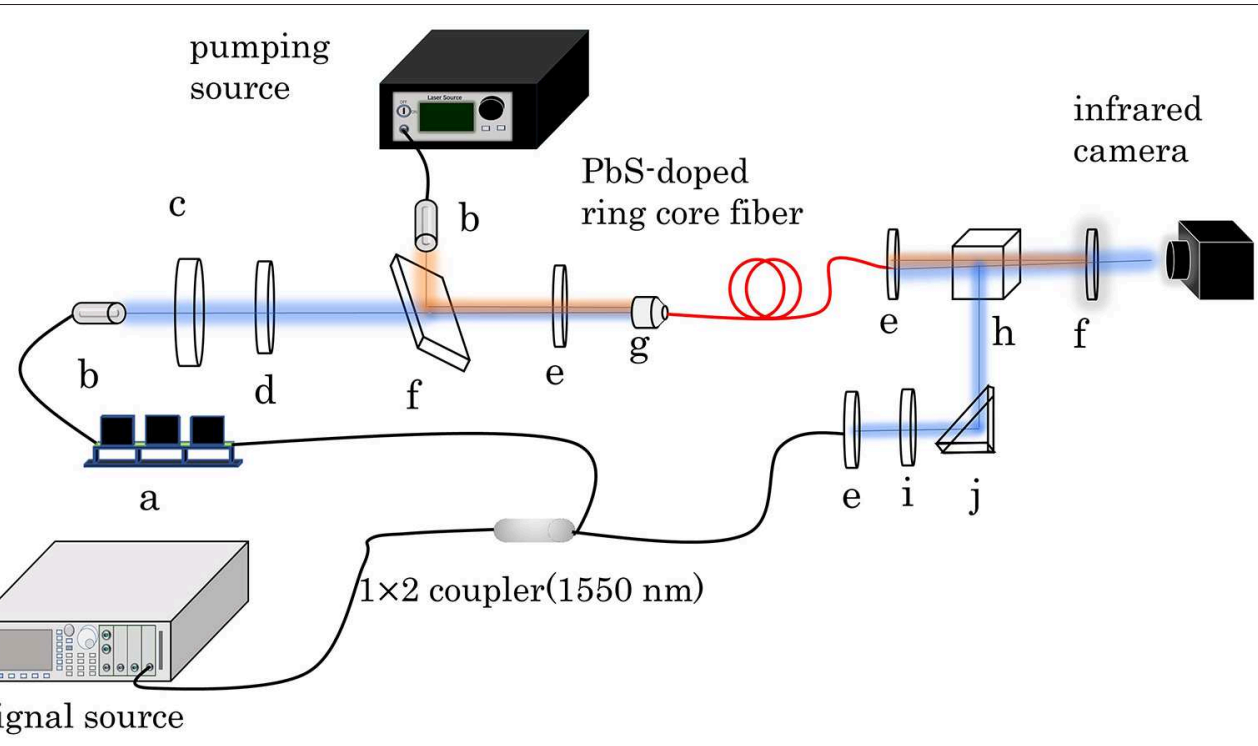

FIGURE 4 | Setup of the OAM amplifier [a, polarization controller; b, collimator; c, spiral phase plate; d, quarter-wave plate; e, lens; f, dichroic mirror (DM); g, objective; h, beam-splitter; i, linear polarizers; j, right-angle prism mirror].
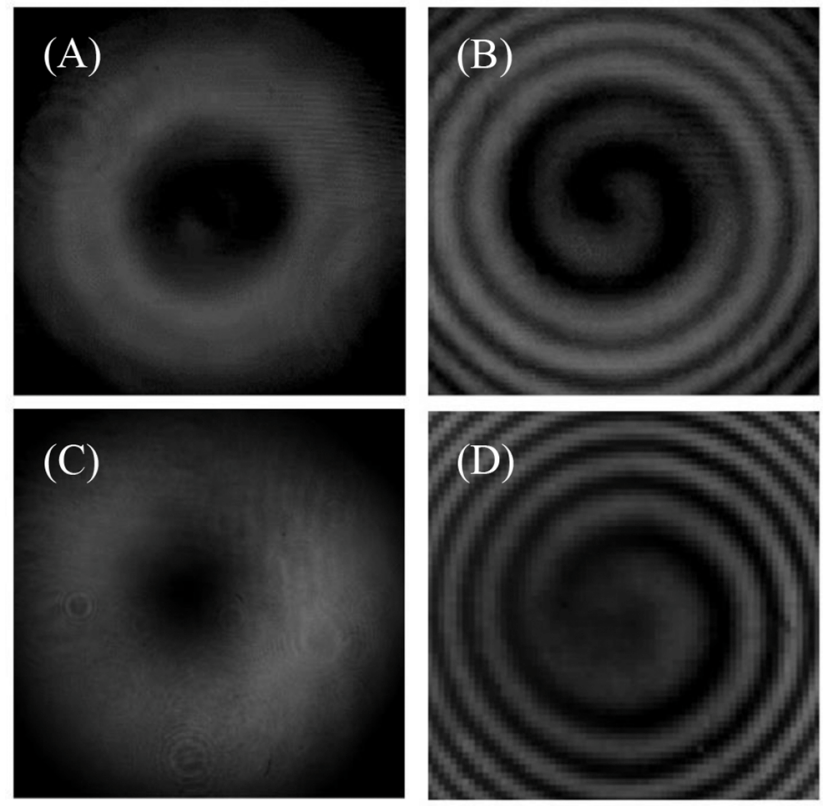

FIGURE 5 | (A) Beam profile before entering the input side of the fiber. (B) Interference before entering the input side of the fiber. (C) Beam profile after exiting the output side of the fiber. (D) Interference after exiting the output side of the fiber.

The on-off gain of a fiber is defined as the ratio of the signal output to input power $[32,33]$. In this paper, the signal power without the pump is taken as the input power and the signal power with the pump as the output power. The gray values measured by the near-infrared camera under different pump powers responses the signal intensity. The same test area (the strip is depicted as a green line in Figure 6A) is used throughout the experiment so that the on-off gain of the optical fiber could be obtained from the gray values. To analyze the on-off gain of the ring-core PbS-doped fiber, the intensity is measured across a strip passing through the center depicted as a green line in Figure 6A. The intensity values gradually increased as the pump power increased from 0 to $750 \mathrm{~mW}$. The on-off gains could be calculated from the intensities. The on-off gains of the OAM $|l|=1$ modes at various pump powers are shown in Figure 6B. The on-off gain of the $\mathrm{OAM}_{11}$ mode reaches $2.3 \mathrm{~dB}$ when the pump power is $750 \mathrm{~mW}$. The small on-off gain of the fiber is mainly attributed to the low $\mathrm{PbS}$ doping concentration.

\section{CONCLUSION}

We experimentally doped the $\mathrm{PbS}$ into a ring-core fiber by using the MCVD method to realize the amplification of the $\mathrm{OAM}_{11}$ mode. The refractive index difference between the cladding and ring core of the fiber is $1 \%$. The fluorescence spectrum of the fiber ranges from 1,100 to $1,600 \mathrm{~nm}$, which is considerably larger than that of erbium-doped fibers and indicates that the fiber can be applied in wide-band amplification systems. This fiber not only supported the transmission of the $\mathrm{OAM}_{11}$ mode but also amplified the mode. The on-off gain of the $\mathrm{OAM}_{11}$ reaches up to $2.3 \mathrm{~dB}$ at $750 \mathrm{~mW} 980 \mathrm{~nm}$ pumping. The experiment can be optimized in the following two aspects to extend the applicability of the fiber to higherorder OAM modes: Firstly, a spatial light modulator can be used to replace the helical phase plate, so that the order of the OAM modes generated can be adjusted by the spatial light modulator. Secondly, increasing the refractive index difference between the core and the cladding appropriately can enhance 

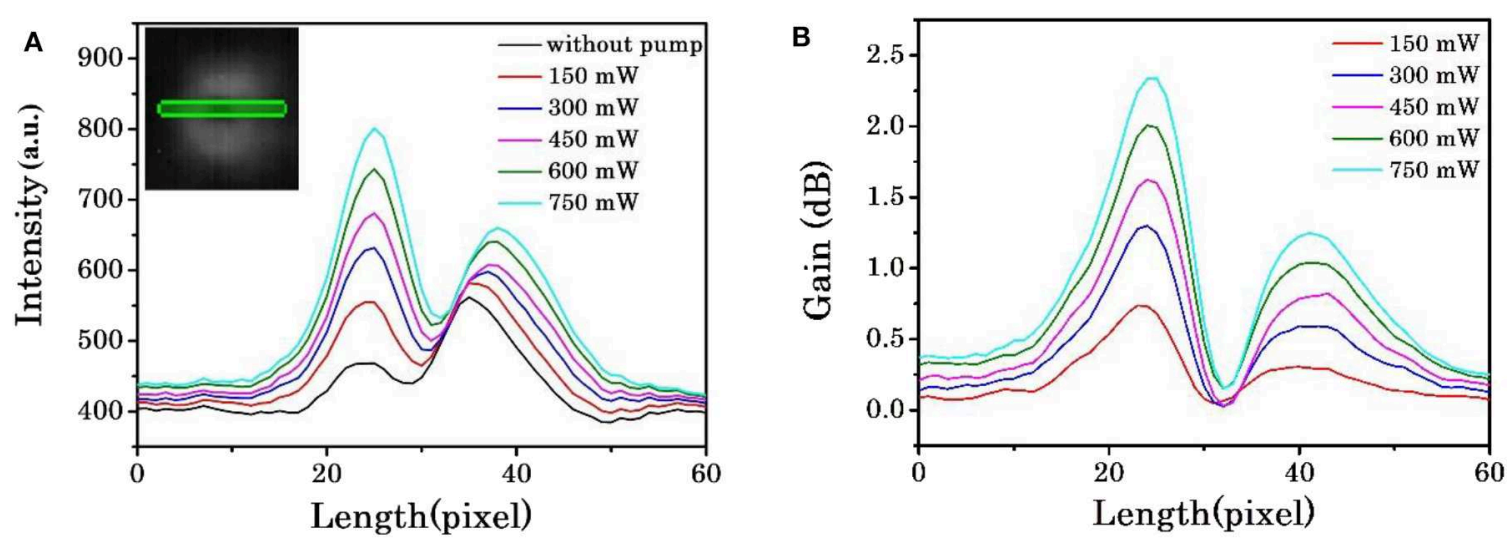

FIGURE 6 | (A) Intensity on the green line. (B) On-off gain on the green line.

the effective refractive index difference of the OAM modes and increase the number of OAM modes transmitted. In addition, the gain can be enhanced by optimizing the fiber fabrication technology to increase the $\mathrm{PbS}$ concentration. This type of PbSdoped ring-core optical fiber amplifier provides the possibility of realizing ultra-wideband OAM mode amplification in future all-fiber transmission systems.

\section{DATA AVAILABILITY STATEMENT}

All datasets generated for this study are included in the article/supplementary material.

\section{AUTHOR CONTRIBUTIONS}

FP and YS contributed to the conception of the study. LX contributed to conceive and design the experiments, analyzed the

\section{REFERENCES}

1. Jung Y, Lim EL, Kang Q, May-Smith TC, Wong NHL, Standish R, et al. Cladding pumped few-mode EDFA for mode division multiplexed transmission. Opt Express. (2014) 22:29008-13. doi: 10.1364/OE.22.029008

2. Jain S, Jung Y, May-Smith TC, Alam SU, Sahu JK, Richardson DJ. Few-mode multi-element fiber amplifier for mode division multiplexing. Opt Express. (2014) 22:29031-6. doi: 10.1364/OE.22.029031

3. Gibson G, Courtial J, Padgett MJ, Vasnetsov M, Pas'ko V, Barnett SM, et al. Free-space information transfer using light beams carrying orbital angular momentum. Opt Express. (2004) 12:5448-56. doi: 10.1364/OPEX.12.005448

4. Ramachandran S, Kristensen P, Yan MF. Generation and propagation of radially polarized beams in optical fiber. Opt Lett. (2009) 34:25257. doi: 10.1364/OL.34.002525

5. Golowich SE, Kristensen P, Bozinovic N, Gregg P, Ramachandran S. Fibers supporting orbital angular momentum states for information capacity scaling. In: Frontiers in Optics. Rochester, NY (2012). p. FW2D.

6. Huang G, Zhou S, Qin Y, Xue L, Man Y. Modal characteristics analysis of orbital angular momentum in a modified ring fiber. Laser Optoelectron Progress. (2015) 52:080604. doi: 10.3788/LOP52.080604

7. Ramachandran S, Gregg P, Kristensen P, Golowich SE. On the scalability of ring fiber designs for OAM multiplexing. Opt Express. (2015) 23:372130. doi: 10.1364/OE.23.003721 data, and wrote the manuscript. LX, JY, YD, and ZC contributed to perform the experiments. HL, NC, JW, and TW helped perform the analysis with constructive discussions.

\section{FUNDING}

This project was funded by National key research and development program of China (2018YFB1801800) and the program of National Natural Science Foundation of China (61675125, 61875118, 61635006, 61705126, and 61975113). The project was also supported by 111 Project (D20031).

\section{ACKNOWLEDGMENTS}

We would like to thank Editage (www.editage.cn) for English language editing.

8. Brunet C, Ung B, Wang L, Messaddeq Y, LaRochelle S, Rusch LA. Design of a family of ring-core fibers for OAM transmission studies. Opt Express. (2015) 23:10553-63. doi: 10.1364/OE.23.010553

9. Gregg P, Kristensen P, Ramachandran S. OAM stability in fiber due to angular momentum conservation. In: Conference on Lasers and Electro-Optics. San Jose, CA (2014). SM2N.2. doi: 10.1364/CLEO_SI.2014.SM2N.2

10. Bozinovic N, Kristensen P, Ramachandran S. Are orbital angular momentum (OAM/Vortex) states of light long-lived in fibers? In: FIO/LS: Technical Digest. San Jose, CA (2011). p. LWL3.

11. Bozinovic N, Yue Y, Ren Y, Tur M, Kristensen P, Huang H, et al. Terabitscale orbital angular momentum mode division multiplexing in fibers. Science. (2013) 340:1545-8. doi: 10.1126/science.1237861

12. Ma J, Xia F, Li S, Wang J. Design of orbital angular momentum (OAM) erbium doped fiber amplifier with low differential modal gain. In: Optical Fiber Communications Conference and Exhibition (OFC). Los Angeles, CA (2015). p. W2A.40.

13. Kang Q, Gregg P, Jung Y, Lim EL, Shaif-Ul A, Ramachandran S, et al. Amplification of 12 OAM states in an air-core EDF. In: Optical Fiber Communications Conference and Exhibition (OFC). Los Angeles, CA (2015). p. Tu3C.2.

14. Liu J, Wang H, Chen S, Zheng S, Zhu L, Wang A, et al. Demonstration of orbital angular momentum (OAM) fiber amplifier in data-carrying OAMdivision multiplexing and wavelength-division multiplexing (WDM) system. 
Optical Fiber Communications Conference and Exhibition (OFC). Los Angeles, CA (2017). p. W2A.21.

15. Jung Y, Kang Q, Raghuraman S, Daryl H, Yoo S, Gregg P, et al. Optical orbital angular momentum amplifier based on an air-hole erbium-doped fiber. $J$ Lightw Technol. (2017) 35:430-6. doi: 10.1109/JLT.2017.2651145

16. Xing J, Wen J, Wang J, Pang F, Chen Z, Liu Y, et al. All-fiber linear polarization and orbital angular momentum modes amplifier based on few-mode erbium-doped fiber and long period fiber grating. Chinese Opt Lett. (2018) 16:100604. doi: 10.3788/COL201816. 100604

17. Jiangjiang F, Xiaohui L, Zhaojiang S, Chuang Z, Xuwei L, Deying L, et al. 2D ductile transition metal chalcogenides (TMCs): novel highperformance Ag2S nanosheets for ultrafast photonics. Adv Opt Mater. (2020) 8:1901762. doi: 10.1002/adom.201901762

18. Liu J, Li X, Guo Y, Qyyum A, Shi Z, Feng T, et al. SnSe2 nanosheets for subpicosecond harmonic mode-locked pulse generation. Small. (2019) 15:1902811. doi: 10.1002/smll.201902811

19. Zhao Y, Guo P, Li X, Jin Z. Ultrafast photonics application of graphdiyne in the optical communication region. Carbon. (2019) 149:336-41. doi: 10.1016/j.carbon.2019.04.075

20. Liu C, Heo J. Lead chalcogenide quantum dot-doped glasses for photonic devices. Int J Appl Glass Sci. (2013) 4:163-73. doi: 10.1111/ ijag. 12032

21. Tong X, Zhou Y, Jin L, Basu K, Adhikari R, Selopal GS, et al. Heavy metal-free, near-infrared colloidal quantum dots for efficient photoelectrochemical hydrogen generation. Nan Ene. (2017) 31:4419. doi: 10.1016/j.nanoen.2016.11.053

22. Zhang Y, Li X, Qyyum A, Feng T, Guo P, Jiang J, et al. PbS nanoparticles for ultrashort pulse generation in optical communication region. Particle Particle Syst Charact. (2018) 35:1800341. doi: 10.1002/ppsc.201800341

23. Zhang Y, Li X, Qyyum A, Feng T, Guo P, Jiang J. Lead sulfide nanoparticles for dual-wavelength ultrashort pulse generation. Nanotechnology. (2019) 31:085202. doi: 10.1088/1361-6528/ab53b4

24. Wundke K, Auxier J, Schülzgen A, Peyghambarian N, Borrelli NF. Roomtemperature gain at $1.3 \mu \mathrm{m}$ in PbS-doped glasses. Appl Phys Lett. (1999) 75:3060-2. doi: 10.1063/1.125230

25. Heo J, Liu C. Pbs quantum-dots in glass matrix for universal fiber-optic amplifier. J Mater Sci Mater Electron. (2007) 18:1359. doi: 10.1007/s10854-007-9172-1
26. Pang F, Sun X, Guo H, Yan J, Wang J, Zeng X, et al. A PbS quantum dots fiber amplifier excited by evanescent wave. Opt Express. (2010) 18:1402430. doi: 10.1364/OE.18.014024

27. Wu Y, Shang Y, Kang Y, Pang F, Wen J, Chen N, et al. Tapered optical fiber deposited with $\mathrm{PbS}$ as an optical fiber amplifier based on atomic layer deposition. Opt Eng. (2018) 57:1. doi: 10.1117/1.OE.57.6.066102

28. Shen K, Baig S, Jiang G, Paik Y-h, Kim SJ, Wang MR. Improved light emitting $\mathrm{UV}$ curable $\mathrm{PbS}$ quantum dots-polymer composite optical waveguides. Opt Commun. (2017) 402:606-11. doi: 10.1016/j.optcom.2017.06.083

29. Cheng C, Wu C. PbS quantum-dot-doped fiber amplifier in NIR S-C-L ultra-broad waveband with low noise. Acta Opt Sin. (2018) 38:1006006. doi: 10.3788/AOS201838.1006006

30. Shang Y, Wen J, Dong Y, Zhan H, Luo Y, Peng G, et al. Luminescence properties of $\mathrm{PbS}$ quantum-dot-doped silica optical fibre produced via atomic layer deposition. J Lumin. (2017) 187:201-4. doi: 10.1016/j.jlumin.2017.03.009

31. Huang X, Fang Z, Kang S, Peng W, Dong G, Zhou B, et al. Controllable fabrication of novel all solid-state $\mathrm{PbS}$ quantum dot-doped glass fibers with tunable broadband near-infrared emission. J Mater Chem C. (2017) 5:792734. doi: 10.1039/C7TC02623D

32. Zheng J, Dong Y, Pan X, Wen J, Chen Z, Pang F, et al. Ultra-wideband and flat-gain optical properties of the PbS quantum dots-doped silica fiber. Opt Express. (2019) 27:37900-9. doi: 10.1364/OE.27.037900

33. Rosolem JB, Horiuchi MR, de Barros MRX, Rocha ML, dos Santos MAD, Juriollo AA. L-band raman amplifier pumped by erbium-doped fiber laser for DS fiber loss compensation. Opt Ampl Appl. (2001) OTuE4. doi: 10.1364/OAA.2001.OTuE4

Conflict of Interest: The authors declare that the research was conducted in the absence of any commercial or financial relationships that could be construed as a potential conflict of interest.

Copyright (c) $2020 \mathrm{Xu}$, Shang, Yang, Chen, Pang, Liu, Dong, Chen, Wen and Wang. This is an open-access article distributed under the terms of the Creative Commons Attribution License (CC BY). The use, distribution or reproduction in other forums is permitted, provided the original author(s) and the copyright owner(s) are credited and that the original publication in this journal is cited, in accordance with accepted academic practice. No use, distribution or reproduction is permitted which does not comply with these terms. 